\title{
Dual-channel fiber surface plasmon resonance biological sensor based on a hybrid interrogation of intensity and wavelength modulation
}

Lixia Li

Xinpu Zhang

Yuzhang Liang

Jianye Guang

Wei Peng 


\title{
Dual-channel fiber surface plasmon resonance biological sensor based on a hybrid interrogation of intensity and wavelength modulation
}

\author{
Lixia Li, Xinpu Zhang, Yuzhang Liang, Jianye Guang, and Wei Peng* \\ Dalian University of Technology, College of Physics and Optoelectronic Engineering, Linggong Road No. 2, Dalian 116024, China
}

\begin{abstract}
We investigate an intensity and wavelength modulation combined plasmon resonance-based fiberoptic sensor technology. Composed of gold nanoparticles (GNPs) and sandwich configuration of Au/indium tin oxide (ITO)/Au film, two sensing regions are fabricated separately along with unclad portions of the fiber-optic probe. It can simultaneously monitor both the light intensity from the Au NP channel and the wavelength from the $\mathrm{Au} / \mathrm{ITO} / \mathrm{Au}$ film channel with a single detector. As the refractive index (RI) of the external environment changes, the transmission intensity and resonance wavelength in the two channels are modified, which provides an interrogation of intensity and wavelength modulation. The sandwich film structure is formed using magnetron sputtering technology, and the GNPs functioning as localized surface plasmon resonators are coated on a multimode optical fiber via the layer-by-layer method. The experimental results reveal that the $\mathrm{RI}$ sensitivities of the two sensing channels are $334.1 \% \mathrm{RIU}^{-1}$ and $1963.2 \mathrm{~nm} / \mathrm{RIU}$, respectively. Based on the above sensing design, we conduct real-time and label-free monitoring of $\mathrm{lgG} / \mathrm{anti}-\mathrm{lgG}$ and Con A/RNase B biomolecular interaction. The resonant dips excited by different sensing modes make it more attractive as a multichannel surface plasmon resonance analysis technology, which is valuable in biological and life sciences research and rapid diagnostics. ๑) 2016 Society of Photo-Optical Instrumentation Engineers (SPIE) [DOI: 10.1117/1.JBO.21.12.127001]
\end{abstract}

Keywords: optical fiber sensor; dual channel; surface plasmon resonance; biological sensing and sensors.

Paper 160487RR received Jul. 14, 2016; accepted for publication Nov. 16, 2016; published online Dec. 8, 2016.

\section{Introduction}

In recent years, surface plasmon resonance (SPR) has been widely explored for various sensing applications. ${ }^{1-3}$ Among all SPR technologies, localized SPR (LSPR) of metal nanoparticles (NPs) has attracted much attention. ${ }^{4-8}$ Similar to SPR, LSPR is the collective electron oscillations of metal NPs when the incident photon frequency conforms to the oscillation frequency of conductive electrons, but its frequency can be tuned by changing the size, geometry, material, and surrounding dielectrics of the NPs. Currently, most fiber-optic SPR sensors are designed for singlechannel detection, ${ }^{9-11}$ which cannot detect more than one analyte or eliminate the adverse effect caused by the changes in the refractive index (RI) of the bulk solution. Seldom do sensors have multiple sensing areas fabricated on a short section of optical fiber, which can realize multichannel detection and self-compensation. ${ }^{12}$ Various approaches have been developed for obtaining two sensing channels on a single optical fiber. ${ }^{13,14}$ For example, a dual-channel SPR probe is formed by coating a polymer layer on gold film to tune the SPR resonance wavelength approximately $100 \mathrm{~nm} .{ }^{15}$ It uses wavelength interrogation for multiple wavelengths but cannot detect changes in the RI of the bulk solution induced by the presence or absence of biochemical molecules. A two-cascaded LSPR fiber sensor based on intensity interrogation has been demonstrated to simultaneously detect two analytes. ${ }^{16}$ However, intensity interrogation is susceptible to stability of the light source, and its fabrication process is relatively complex. Other SPR sensors based on fiber-optic bundles ${ }^{17}$ to realize multichannel detection limit the miniaturization of the sensing element, and the procedure is complex. Rapid and parallel detection in a single chip based on prism-configuration SPR and LSPR spectroscopy have been realized previously, but they always depend on complex and expensive components, which are not appropriate in practice for structure miniaturization. ${ }^{18-20}$

In this paper, we proposed a dual-channel fiber-optic sensing probe based on two sensing structures with different modulation methods (intensity and wavelength interrogation). Two unclad portions of the fiber probe are coated with gold nanoparticles (GNPs) and a sandwich configuration of Au/indium tin oxide (ITO)/Au film, respectively. By depositing a thin high-index ITO dielectric layer into the gold-sensing surface, the SPR resonance wavelength can be modulated. We can tune the transmission intensity of the intensity interrogation-based channel ( $\mathrm{Au}$ NP channel) and resonance wavelength of the wavelength interrogation-based channel (Au/ITO/Au film channel) by modifying the surrounding RI, which creates a dual-channel hybrid intensity-wavelength-modulated SPR sensor probe. The assay time for a kind of biomolecular sample detection is less than $20 \mathrm{~min}$, and it can broaden the SPR detection range of a sensor from visible to near infrared. Furthermore, the performance of the sensor is comparable with traditional SPR sensors. ${ }^{21,22}$

\section{Materials and Methods}

As depicted in Fig. 1(a), light from a halogen lamp (HL-2000FHSA, Ocean Optics, Inc.) was launched into one end of the probe. The transmission spectrum was collected by a spectrometer (HR4000, Ocean Optics, Inc.) and demodulated by a 


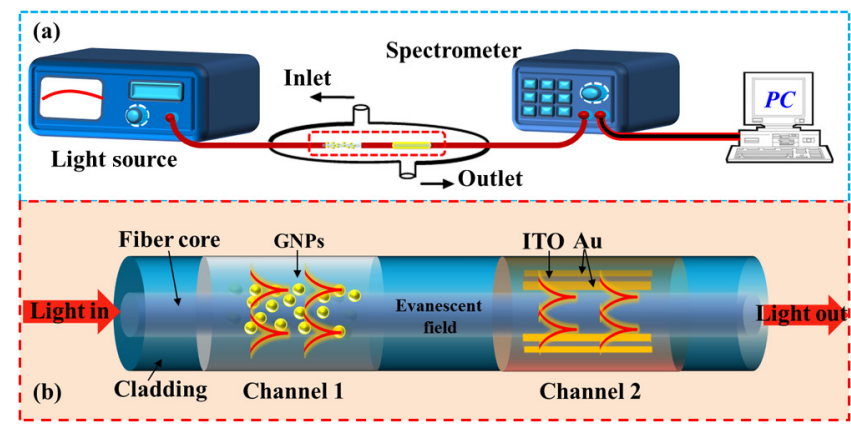

Fig. 1 Schematics of dual-channel fiber-optic SPR sensor system based on GNPs and Au/ITO/Au coatings to simultaneously realize intensity and wavelength modulation. (a) A sketch of the experimental setup for sensing measurement. (b) A dual-channel SPR sensing probe. Channel 1 is composed of GNPs, and Channel 2 is built with $\mathrm{Au} / \mathrm{ITO} / \mathrm{Au}$.

computer. We used a home-built flow cell to control the sample solution insertion and ejection. For comparison, a $1.0 \mathrm{ml} / \mathrm{min}$ flow rate controlled by a peristaltic pump was selected for injecting the analytes. Figure 1(b) illustrates the schematic diagram of the two channels of the probe. The fiber probes were prepared using multimode plastic-clad silica-optical fiber (0.37 NA, $400-\mu \mathrm{m}$ core, and $430-\mu \mathrm{m}$ cladding diameter). The two end faces of probe were polished to decrease connection loss between the probe and fiber jumper. The two unclad core sections of optical fiber were cleaned with deionized water and acetone and dried with a pure $\mathrm{N}_{2}$ gas stream. Then, the two sections were coated with 40-nm gold particles and a 35-nm 35-nm $\mathrm{Au} / 10-\mathrm{nm}$ ITO/15-nm Au layer, respectively. They were labeled as Channel 1 and Channel 2. In Channel 1, the self-synthesized spherical gold $\mathrm{NPs}^{23}$ were immobilized on the core of the fiber by utilizing the layer-by-layer method. The specific preparation process of Channel 1 was as follows: the core of optical fiber was first immersed in Piranha solution (a mixture consisting of seven volumes of concentrated $\mathrm{H}_{2} \mathrm{SO}_{4}$ and three volumes of $30 \% \mathrm{H}_{2} \mathrm{O}_{2}$ ) for $45 \mathrm{~min}$, followed by thorough flushing with deionized water, and dried with a pure $\mathrm{N}_{2}$ gas stream; then, a trilayer polyelectrolyte (PE) structure was selected for selfassembly of GNPs. The channel was sequentially dipped in poly(diallyldimethylammonium chloride) (strong cationic $\mathrm{PE}$ ), poly(sodium- $p$-styrenesulfonate) (the anionic PE), and poly(allylamine hydrochloride) (PAH, weak cationic PE). The soak time for etch PE was 15 min. Finally, the GNPs stabilized by citrate were immobilized on the outermost PAH layer for $120 \mathrm{~min}$ to form a self-assembled monolayer. In our test, the NP self-assembled monolayer was extremely stable during the six repeated binding and regeneration conditions. In Channel 2, the Au/ITO/Au film was coated on the cleaned unclad core using the Turbo Sputter Coater (K575XD from E. M. Technologies Ltd. Ashford, Kent).

\section{Results and Discussion}

The response characteristics of the RI of the dual-channel fiberoptic sensor were experimentally investigated. Figure 2(a) presents the transmission spectra of the proposed biosensor versus RIs. As the surrounding RI increases gradually, the transmission intensity of the dip A corresponding to Channel 1 decreases significantly and the dip wavelength has negligible shifts. Additionally, the wavelengths of dip B corresponding to Channel 2 increase while the surrounding RI increases from 1.3266 to 1.3683 . The normalized intensity changes of Channel 1 and the wavelength shifts of Channel 2 both have a linear response to the bulk RI between 1.3266 and 1.3683 ,

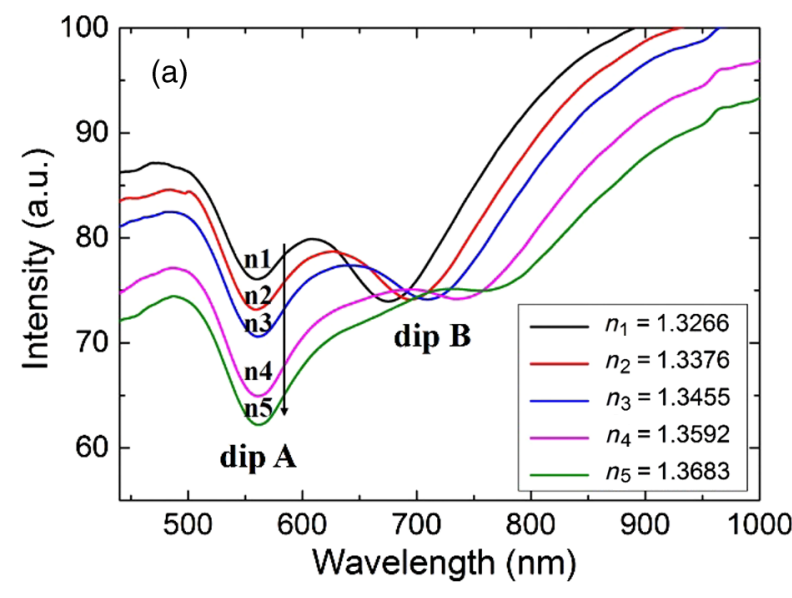

Fig. 2 Rl response of the dual-channel fiber-optic senso the proposed biosensor for sodium chloride solutions with different Rls. (b) The relationship of sample RI and measured relative intensity of dip A in Channel 1. (c) The relationship of sample RI and measured wavelength of dip B in Channel 2.
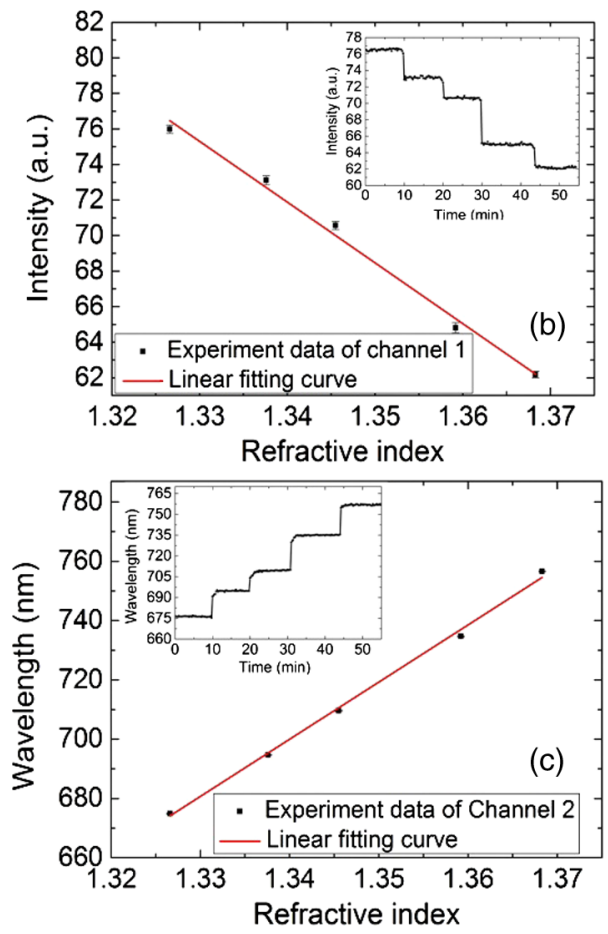
which is consistent with the range for the most common biofluids, as shown in Figs. 2(b) and 2(c). The corresponding RI sensitivities $S$ are $334.1 \% \mathrm{RIU}^{-1}$ and $1963.2 \mathrm{~nm} / \mathrm{RIU}$ for the GNPs and the Au/ITO/Au film channels, respectively. Here, RI sensitivity $S$ is defined as the change of resonant wavelength $(S=\Delta \lambda / \Delta n)$ and resonant intensity $(S=\Delta \lambda / \Delta n)$ with respect to the bulk RI changes. The resolution $R$ of the RI measurement is estimated to be $5.9 \times 10^{-5}$ RIU and $5.09 \times 10^{-6}$ RIU, respectively, which is defined as $R=N / S$, where $N$ is the standard deviation of noise and $S$ is the RI sensitivity. $N$ can be calculated from the time response of the resonant dip for the fixed RI of the bulk solution.

To further understand the operating principle of the dualchannel SPR sensor, Fig. 3 shows the distribution of the spatial electric field of different resonance positions caused by different resonance modes. The black dotted lines in Figs. 3(c) and 3(e) represent the boundary of the $\mathrm{Au} / \mathrm{ITO} / \mathrm{Au}$ composite and $\mathrm{Au}$ films, respectively. As depicted in Figs. 3(a), 3(c), and 3(e), the electric field is mostly confined and the maximum field distribution is exhibited on the surface of the NPs or metal film. Figures 3(b), 3(d), and 3(f) illustrate the transmission spectra of LSPR and SPR modes, respectively. It can be seen that the LSPR resonance dip caused by GNP absorption appears in the range of 500 to $600 \mathrm{~nm}$. Simultaneously, there is an obvious dip located at the range of 600 to $1000 \mathrm{~nm}$, induced by the Au/ITO/Au film channel. As shown in Figs. 3(c) and 3(e), compared with the pure gold layer SPR structure, the composited $\mathrm{Au} / \mathrm{ITO} / \mathrm{Au}$ layer structure with the same thickness shows a stronger near-field intensity enhancement (the field intensity maximum on the surface of the composited $\mathrm{Au} /$ ITO/Au layer structure is 3.8, whereas that of the Au layer is 3.3), which is beneficial to improve the interaction between the evanescent field and biomolecules and achieve highprecision detection. Furthermore, to avoid crosstalk between the channels (the resonance wavelengths of the different channels are located at different spectral positions), the SPR wavelengths
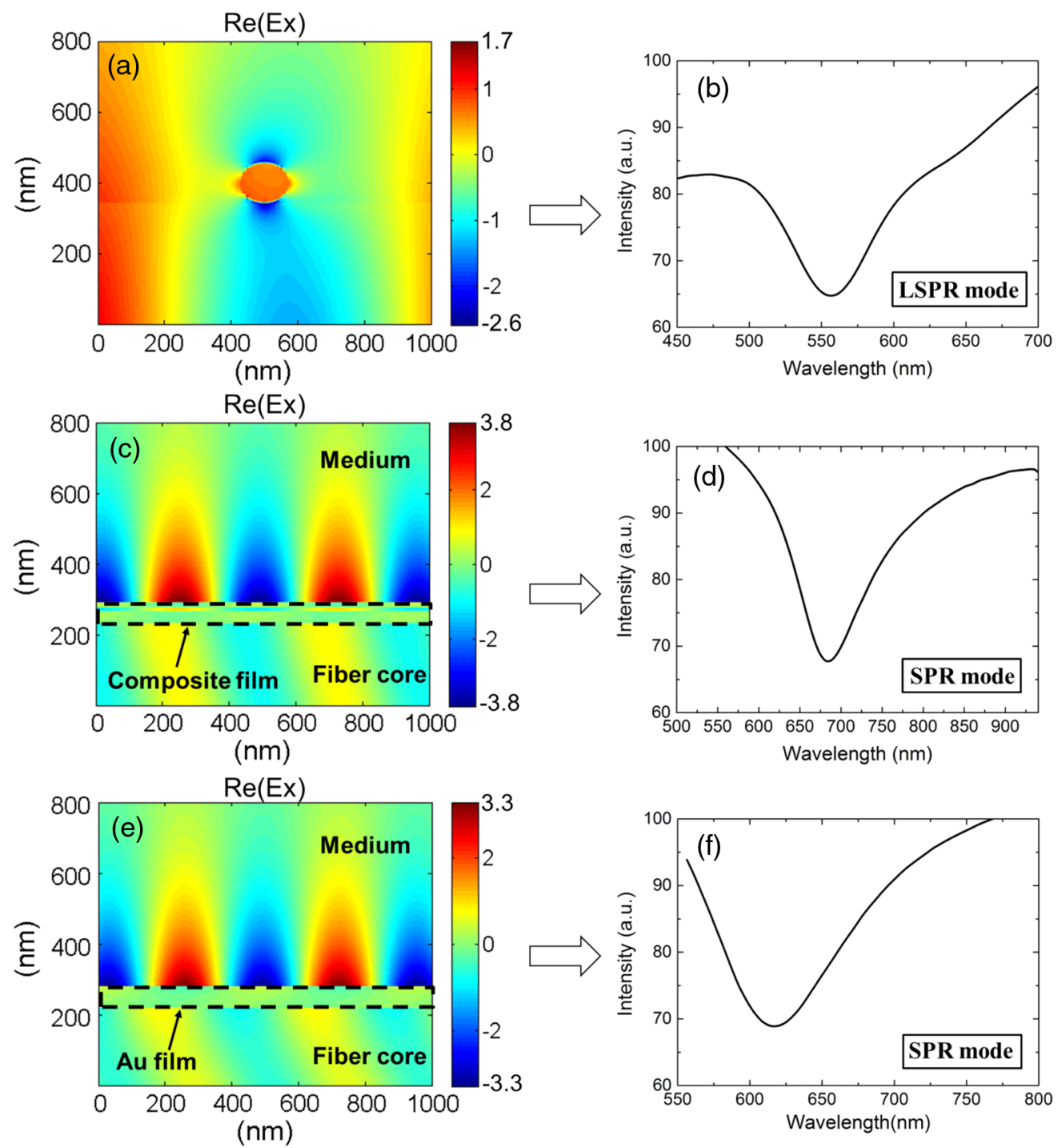

Fig. 3 Spatial electric field analysis of the dual modulation-based fiber-optic sensor. (a) Real part distribution of spatial electric field $(|\mathrm{Ex}|)$ and (b) transmission spectrum of LSPR mode for resonant position at $560 \mathrm{~nm}$. (c) Real part distribution of spatial electric field (|Ex|) and (d) transmission spectrum of SPR mode (35-nm Au/10-nm ITO/15-nm Au layer) for resonant position at $678 \mathrm{~nm}$. (e) Real part distribution of spatial electric field $(|\mathrm{Ex}|)$ and (f) transmission spectrum of SPR mode (60-nm Au layer) for resonant position at $620 \mathrm{~nm}$. 
of the channels should be able to tune over a wide range to completely separate the two channels. There are several approaches to realize the change of the operation wavelength of the SPR sensor, ${ }^{24,25}$ and some of them are too complex. In order to simplify the fabrication, a thin layer of ITO was selected to deposit between gold layers to form the sandwich configuration. As a high-RI material, the ITO coating is one of the ideal candidates to tune the operating wavelength of the SPR sensor. Fig. 3(d) shows that the SPR resonance wavelength shifts toward the longer wavelength when we use the Au/ITO/Au sandwich configuration.

To further investigate the multianalyte sensing feasibility of the dual modulation (intensity and wavelength interrogation) of the dual-channel sensor, we carried out model experiments in a buffer solution to kinetically monitor the molecular recognition events. Anti-rabbit immunoglobulin G (anti-IgG) was functionalized on the GNP channel as the molecular recognition probe to specific binding with $\mathrm{IgG}$, and ribonuclease B (RNase B) was fixed on the Au/ITO/Au film channel as the recognition part to specific binding with Con A. The immobilizing processes of IgG and Con A are depicted in Fig. 4. We immersed two channels in 11-mercaptoundecanoic acid $(1 \mathrm{mmol} / \mathrm{L})$ at room temperature for $12 \mathrm{~h}$ to self-assemble an alkanethiol monolayer on the surfaces; then the probe was immersed in an aqueous solution containing 1-ethyl-3-(3-dimethylamino-propyl) carbodiimide hydrochloride $(0.55 \mathrm{~mol} / \mathrm{L})$ and $N$-hydroxysuccinimide $(0.5 \mathrm{~mol} / \mathrm{L})$ for $30 \mathrm{~min}$ at $4^{\circ} \mathrm{C}$ to activate the carboxyl of the alkanethiol layer. After activating, the probe was rinsed with deionized water and dried under nitrogen. Channel 1 was immersed in anti-rabbit $\mathrm{IgG}$ solution $[0.1 \mathrm{mg} / \mathrm{ml}$ in $0.01 \mathrm{M}$ phosphate-buffered saline buffer (PBS buffer, $\mathrm{pH}=7.4$ )], and Channel 2 was immersed in RNase B solution $(0.1 \mathrm{mg} / \mathrm{ml}$ in $0.01 \mathrm{M}$ PBS buffer, $\mathrm{pH}=7.4$ ) for $30 \mathrm{~min}$ to form a stable monomolecular layer, respectively. After rinsing with PBS

(a)

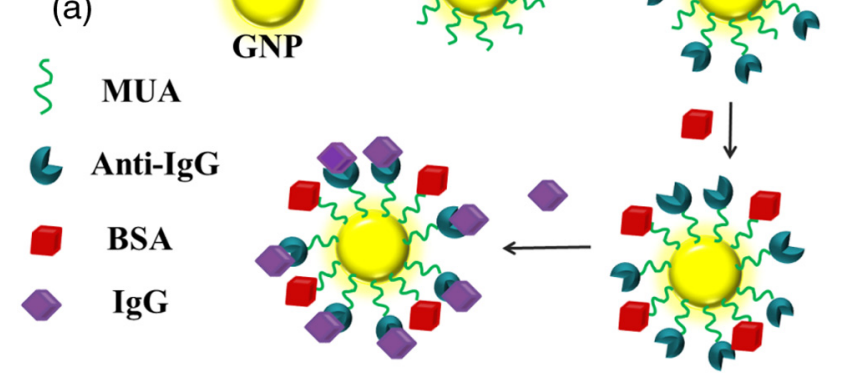

(b)
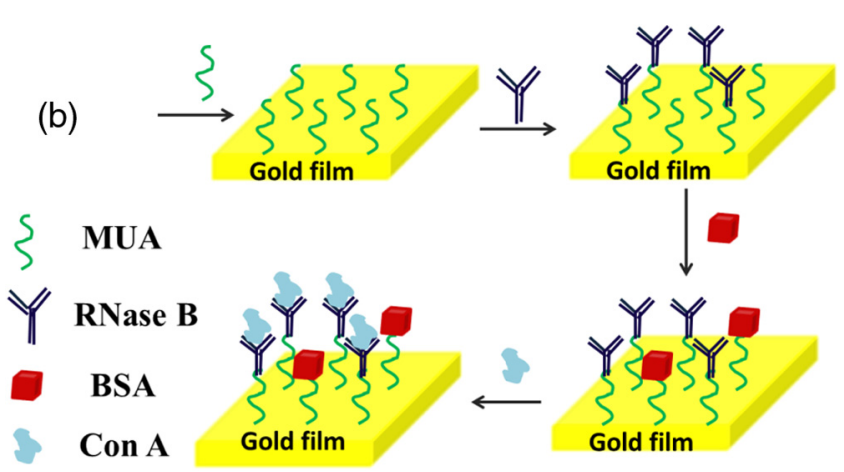

Fig. 4 Processes for specific binding of (a) IgG and (b) Con A. buffer, $0.1 \mathrm{mg} / \mathrm{ml}$ bovine serum albumin was used to deactivate the unreacted groups on the double-channel surfaces. Finally, the functional probe was obtained and could be used for the measurement of specific biomolecular binding.

The biological monitoring performance of this dual-channel fiber biosensor was evaluated based on the specific binding of IgG to anti-IgG and Con A to RNase B. IgG and Con A samples with different concentrations were prepared in a PBS buffer $(\mathrm{pH}=7.4)$. Before the samples were injected into the flow cell, the PBS buffer was introduced to establish the baseline value. The real-time response of the two channels was monitored by LabVIEW, and the experimental data were processed every $2 \mathrm{~s}$. The time response of the specific biomolecular binding of the two channels is shown in Fig. 5. As a comparison, we also gave the signal changes of nonspecific binding in the other channels during IgG and Con A detection in Figs. 5(a) and 5(b). It is worth pointing out that the real-time response of nonspecific binding was almost the same for detecting molecules with different concentrations. Therefore, only one comparison curve is shown in Figs. 5(a) and 5(b). The molecular binding will cause the increase of localized RI on the NP surface. Thus, the intensity of 560-nm incident light reduced with the increase of time, then reached a steady state after $1200 \mathrm{~s}$. Figure 5(a) shows that the intensity of Channel 1 decreased due to the injection of $\mathrm{IgG}$, which was consistent with the fact that $\mathrm{IgG}$ bound specifically to anti-IgG on the sensing region surface. Using urea solution $(8.0 \mathrm{M})$ to regenerate the biosensor surfaces, IgG samples with various concentrations ranging from 0.05 to $0.4 \mathrm{mg} / \mathrm{ml}(0.05,0.1,0.2$, and $0.4 \mathrm{mg} / \mathrm{ml})$ were evaluated. The response increased with the increase of IgG concentration. However, the resonance wavelength of Channel 2 remains unchanged, which means no molecular interaction occurred in Channel 2 during $\operatorname{IgG}$ detection. Moreover, the response of Channel 2 also demonstrates there was no significant bulk RI change as the $\operatorname{IgG}$ concentration increased. Figure 5(c) shows the relationship between the intensity of Channel 1 and different concentrations of IgG samples. The normalized intensity decreased linearly with the concentration of the samples. Error bars in Fig. 5 give the variance of three repeated experiments.

Subsequently, the real-time responses of the designed biosensor to Con A with different concentrations (0.05, 0.08, $0.1,0.3$, and $0.5 \mathrm{mg} / \mathrm{ml}$ ) were experimentally investigated. As shown in Fig. 5(b), the resonance wavelength of Channel 2 had a red-shift due to the bonding between Con $\mathrm{A}$ and RNase B. Likewise, the unchanged intensity of Channel 1 can also indicate there was no significant bulk RI change during the Con A detection process. The relationship between different concentrations of Con A samples and wavelength response of Channel 2 is shown in Fig. 5(d). The resonance wavelength moved toward longer wavelengths linearly as Con A concentration increased. The experiment of introducing a buffered mixture containing both $0.05 \mathrm{mg} / \mathrm{ml} \mathrm{IgG}$ and $0.05 \mathrm{mg} / \mathrm{ml} \mathrm{Con} \mathrm{A}$ was also performed. The results showed similar intensity and wavelength responses as Figs. 5(a) and 5(b), except that the signal change occurred almost at the same time. Through the utilization of a reference channel, we can ensure that the measured signal change was caused by the specific binding event between analyte and corresponding recognition molecules on the sensing surface. The limits of detection were 0.05 and $0.01 \mathrm{mg} / \mathrm{ml}$ for the two channels, respectively. 

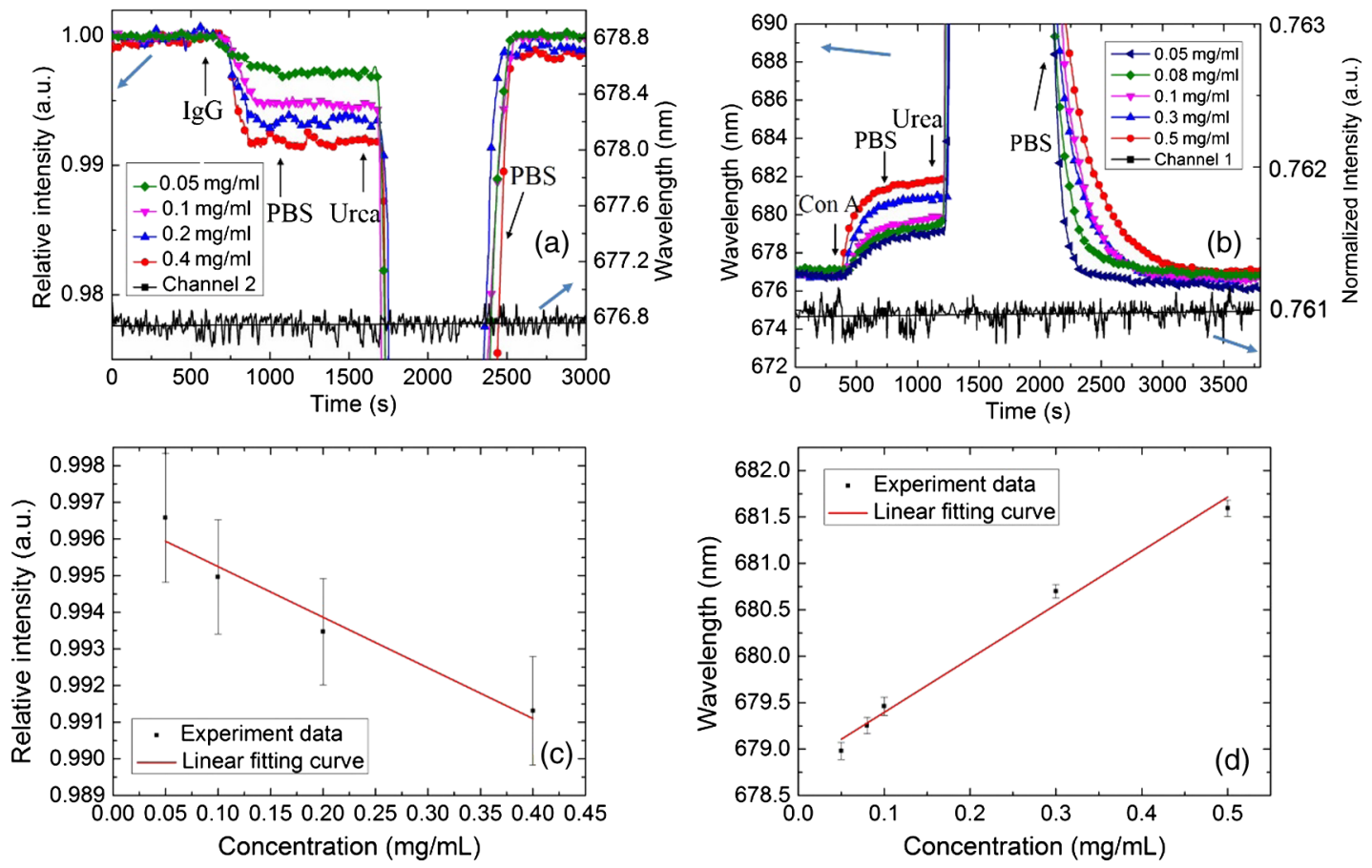

Fig. 5 (a) Response of two channels during IgG detection. (b) Response of two channels during Con A detection. (c) Calibration curve of Channel 1. (d) Calibration curve of Channel 2.

\section{Conclusion}

In summary, we proposed and demonstrated a dual-channel fiberoptic biosensor using a hybrid interrogation of intensity and wavelength modulation. The intensity and wavelength modulation were generated from the SPR of GNPs and a sandwich configuration of $\mathrm{Au} / \mathrm{ITO} / \mathrm{Au}$ film, respectively. We fabricated dualchannel prototypes with a combination of GNPs and the sandwich configuration of $\mathrm{Au} / \mathrm{ITO} / \mathrm{Au}$ film. The sensing technology is completed by measuring the intensity variation and wavelength shift of the resonance dips in the transmission spectrum. In the RI range from 1.3266 to 1.3683 , the corresponding sensitivities are $334.1 \% \mathrm{RIU}^{-1}$ and $1963.2 \mathrm{~nm} / \mathrm{RIU}$ for the GNPs and the $\mathrm{Au} /$ ITO/Au film channels, respectively. We investigated the multianalyte sensing feasibility of this structure when the sensor probes are functionalized by different biological molecules and the dual channels can detect different biochemical targets simultaneously. The biochemical response demonstrates that this sensor can be used for dual-channel measurement and improve the detection efficiency. Future works will be focused on sensitivity and resolution enhancement by optimizing sensor structure and materials.

\section{Disclosures}

No conflicts of interest, financial or otherwise, are declared by the authors.

\section{Acknowledgments}

The authors would like to thank the National Natural Science Foundation of China (61137005 and 11474043) for financial support.

\section{References}

1. A. K. Sharma et al., "Theoretical understanding of an alternating dielectric multilayer-based fiber optic SPR sensor and its application to gas sensing," New J. Phys. 10(2), 023039 (2008).
2. S. Franzen et al., "Plasmonic phenomena in indium tin oxide and ITO-Au hybrid films," Opt. Lett. 34(18), 2867-2869 (2009).

3. R. Verma et al., "Detection of heavy metal ions in contaminated water by surface plasmon resonance based optical fiber sensor using conducting polymer and chitosan," Food Chem. 166, 568-575 (2015).

4. Z. Q. Tou et al., "Fiber optic refractometer based on cladding excitation of localized surface plasmon resonance," IEEE Photonics Technol. Lett. 25(6), 556-559 (2013).

5. H. Y. Lin et al., "Tapered optical fiber sensor based on localized surface plasmon resonance," Opt. Express 20(19), 21693-21701 (2012).

6. J. Cao et al., "Gold nanorod-based localized surface plasmon resonance biosensors: a review," Sens. Actuators, B 195, 332-351 (2014).

7. Y. B. Lin et al., "A reflection-based localized surface plasmon resonance fiber-optic probe for biochemical sensing," Biomed. Opt. Express 2(3), 478-484 (2011)

8. S. Szunerits et al., "Sensing using localised surface plasmon resonance sensors," Chem. Commun. 48(72), 8999-9010 (2012).

9. J. H. Ahn et al., "Fiber-optic waveguide coupled surface plasmon resonance sensor," Opt. Express 20(19), 21729-21738 (2012).

10. C. Perrotton et al., "Fiber optic surface plasmon resonance sensor based on wavelength modulation for hydrogen sensing," Opt. Express 19(106), A1175-A1183 (2011).

11. N. Raoufi et al., "Fiber optic pH sensor using optimized layer-by-layer coating approach," IEEE Sens. J. 14(1), 47-54 (2014).

12. R. Verma et al., "A novel approach for simultaneous sensing of urea and glucose by SPR based optical fiber multianalyte sensor," Analyst 139(6), 1449-1455 (2014).

13. L. X. Li et al., "Dual-channel fiber-optic biosensor for self-compensated refractive index measurement," IEEE Photonics Technol. Lett. 28(19), 2110-2113 (2016).

14. Y. Liu et al., "Investigation of a capillary-based surface plasmon resonance sensor for biosensing," J. Lightwave Technol. 34(17), 4036-4042 (2016).

15. W. Peng et al., "Investigation of dual-channel fiber-optic surface plasmon resonance sensing for biological applications," Opt. Lett. 30(22), 2988-2990 (2005).

16. H. Y. Lin et al., "Multiple resonance fiber-optic sensor with time division multiplexing for multianalyte detection," Opt. Lett. 37(19), 3969-3971 (2012).

17. Y. Liu et al., "Compact multi-channel surface plasmon resonance sensor for real-time multi-analyte biosensing," Opt. Express 23(16), 2054020548 (2015). 
Li et al.: Dual-channel fiber surface plasmon resonance biological sensor based on a hybrid interrogation...

18. S. Mohseni et al., "Development of a label-free SPR sensor for detection of matrixmetalloproteinase-9 by antibody immobilization on carboxymethyldextran chip," Biosens. Bioelectron. 81, 510-516 (2016).

19. K. Tiwari et al., "Surface plasmon based sensor with order-of-magnitude higher sensitivity to electric field induced changes in dielectric environment at metal/nematic liquid-crystal interface," Sens. Actuators, A 216, 128-135 (2014).

20. K. Sathiyamoorthy, "Modified two prism SPR sensor configurations to improve the sensitivity of measurement," Sens. Actuators, A 191, 73-77 (2013).

21. S. M. Chen et al., "Localized surface plasmon resonance-based microcapillary biosensor," IEEE Photonics Technol. Lett. 28(20), 2195-2198 (2016).

22. X. H. Zhao et al., "Optical fiber sensor based on surface plasmon resonance for rapid detection of avian influenza virus subtype H6: initial studies," J. Virol. Methods 233, 15-22 (2016).

23. S. F. Cheng et al., "Colloidal gold-modified optical fiber for chemical and biochemical sensing," Anal. Chem. 75(1), 16-21 (2003).

24. L. L. Obando et al., "Tuning dynamic range and sensitivity of whitelight, multimode, fiber-optic surface plasmon resonance sensors," Anal. Chem. 71(22), 5116-5122 (1999).

25. D. Monzon-Hernandez et al., "Optical-fiber surface-plasmon resonance sensor with multiple resonance peaks," Appl. Opt. 43(6), 1216-1220 (2004).

Lixia $\mathbf{L i}$ received her $\mathrm{BE}$ degree in the School of Physics and Electronic Engineering from Zhengzhou University of Light Industry, Zhengzhou, China, in 2012. She is currently pursuing her PhD in optical engineering at Dalian University of Technology. Her research area is focused on fiber-optic sensors, surface plasmon resonance, and subwavelength optical design and fabrication.
Xinpu Zhang received his $\mathrm{PhD}$ in optical engineering from the Department of Physics at Dalian University of Technology, Dalian, China, in 2016. He joined the School of Information Science and Technology at Southwest Jiaotong University as a lecturer. His research area is focused on optical fiber sensors, surface plasmon resonance, and fiber grating.

Yuzhang Liang received his $\mathrm{PhD}$ in optical engineering from the Department of Physics at Dalian University of Technology, Dalian, China, in 2016. He is currently a postdoctor at Nanjing University. His current research interests include surface plasmon resonance and subwavelength optical design and fabrication.

Jianye Guang received his BS degree in optical information science and technology from Dalian University of Technology, Dalian, China, in 2015 . He is currently a postgraduate in optics engineering at the College of Physics and Optoelectronic Engineering, Dalian University of Technology. His current research interests include surface plasmon resonance and biochemical sensing.

Wei Peng received her doctoral degree in optical engineering from Dalian University of Technology, Dalian, China, in 1999. After spending 4 years in the Center for Photonics Technology within the Department of Electrical Engineering at Virginia Polytechnic Institute and State University as a visiting scholar and postdoctor, she took a professor faculty position at Dalian University of Technology, China, in 2009. Currently, her research interests include fiber-optic sensors, surface plasmon resonance, and subwavelength optical design and fabrication. 